\title{
A Prospective Study of Total Knee Replacement with Minimally Invasive Mid Vastus Approach Showing Early Results
}

ISSN: 2576-8875

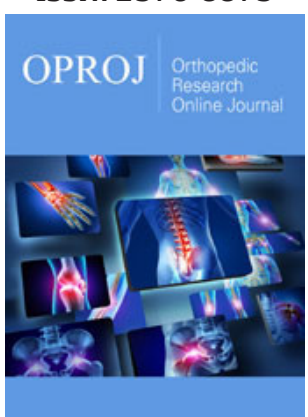

*Corresponding author: Ashish BC, Fellow of Joint replacements and Trauma, Nagpal Superspeciality Hospital, India

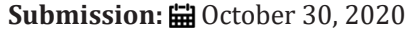

Published: 鹠 November 10, 2020

Volume 7 - Issue 4

How to cite this article: Parampreet S Nagpal, Ashish BC, Sham Goyal. A Prospective Study of Total Knee Replacement with Minimally Invasive Mid Vastus Approach Showing Early Results. Ortho Res Online J. 7(4). OPROJ. 000669. 2020 .

DOI: 10.31031/OPROJ.2020.07.000669

Copyright@: Ashish BC, This article is distributed under the terms of the Creative Commons Attribution 4.0 International License, which permits unrestricted use and redistribution provided that the original author and source are credited.

\author{
Parampreet S Nagpal ${ }^{1}$, Ashish BC ${ }^{2 *}$ and Sham Goyal $^{3}$ \\ ${ }^{1}$ Chief Orthopedic surgeon (Nagpal Superspeciality Hospital), India \\ ${ }^{2}$ Fellow of Joint replacements and Trauma, Nagpal Superspeciality Hospital, India \\ ${ }^{3}$ Department of Anesthesia\& Intensive care, India
}

\begin{abstract}
Objective: The aim of this studythe early results of total knee arthroplasty (TKA)through minimally invasivemidvastus approach.

Methods: This prospective study included 100 knee joints (43 females, 57 males; mean age61.3years) whounder went total knee arthroplasty forgrade 4 knee osteoarthritis.AllTKA operations were performed by the single orthopaedic surgeon through the minimally invasive midvastusapproach.Pre- operatively, all patients had primary osteoarthritis.In all cases,aPScemented prosthesis with modular insertwasused.All the knees were assessed according to theKneeSociety kneeand functions cores before surgery and atthefinal follow-up.Postoperative radiographic evaluations were performed on AP and lateral radiographs according to the knee society scoreevaluation and scoring system. The mean follow-up period was 6 months (range 3 to 12 months).
\end{abstract}

Results: The mean KSS score significantly improved from $27.8 \pm 11.0$ preoperatively to $92.2 \pm 3.8$ post- operatively $(\mathrm{p}=0.000)$.The improvement in the knee functions core was from $54.223 \pm 23.43$ to $94.07 \pm 7.720(\mathrm{p}=0.000)$. The mean improvement in the KSS clinical and functions cores were 64.4 and 39 . 8 points, respectively. The mean knee ROM significantly increased from $56.2 \pm 22.7^{\circ}$ pre- operatively to $111.0 \pm 9.9^{\circ}$ postoperatively $(\mathrm{p}=0.000)$. Patients had nopatellar trackingabnormalityintraoperatively;thus,there was none ed for lateral retinacular release.Postoperative clinical and radio graphicassessments showed no signs of instability or loosing. There were no changes in tracking and loosing of the implant during the follow up period. Neurovascular injury did not occur.

Conclusion: In our study,lateral retinacular release was not doneduetoachievement of proper patellar tracking in TKA with minimally invasive midvastus approach and showing satisfactory clinical and radiographic results

\section{Introduction}

With the advances in science, the life span of people is increasing, leading to a boost in the numbers of the geriatric population. In our country there is an increase in the number of people suffering from osteoarthritis; and also a considerable increase in the Body Mass Index (BMI) which when combined with sitting cross legged and squatting play a strong role in joint degeneration.

This necessitating a surgical intervention with less complications. Total knee replacement has emerged as a more reliable, longer lasting method of knee arthroplasty, with excellent pain relief and functioning. So, this study is intended to do a review of the results of total knee arthroplasty was done with minimally invasive mid vastus approach.

The medial parapatellar approach is the main surgical approach for most of the total knee replacements. Modified approaches such as minimally invasive mid vastus approach have been introduced to improve quadriceps power, improve knee flexion, better pain relief. However, there is a difficulty in patella eversion and visualisation of the lateral tibial plateau as concerns as with reports. Does the minimally invasive mid-vastus approach really provide advantages? We report our prospective study with 100 patients undergoing total knee replacement using a medial mid-vastus approach with one year follow up. 


\section{Materials and Methods}

\section{Aim and objectives}

This study aims to present data on the outcome of total knee replacements done with minimally invasive Mid vastus approach, postoperative functional results and any complication associated with it during the intraoperative procedure. This study was conducted at Nagpal Superspeciality Hospital, Bhatinda, PUNJAB with the follow up period of 12 months from April 2019 to April 2020

\section{Methods}

It is as prospective study where in All the patients were diagnosed with osteoarthritis of knee with the help of knee $\mathrm{x}$ ray AP and lateral views were taken. Knee society score was applied to both pre-operative diagnosis and post-operative scores to evaluate the results

\section{Inclusion criteria:}

a) Age group above 45

b) Diagnosed with osteoarthritis of knee (degenerative, inflammatory or post traumatic arthritis)

c) Unilateral and bilateral knee replacement

d) Patients who have given consent for surgery and to participate in the surgery

\section{Exclusion criteria:}

a) Patients who are medically unfit

b) Patient with extra-articular deformities in knee c) Patient with neuromuscular disorders

d) Patients who have not given consent

\section{Preop planning consists of:}

a) Patients were diagnosed with $\mathrm{OA}$ knee in OPD with both $\mathrm{AP}$ and LT views, Oblique X-rays (if required) were taken

b) Complete blood count, ECG, blood urea and serum creatinine, FBS, HBA1C, HIV and HBSAG profiles, blood grouping was done ECG, 2D-ECHO Where necessary, were done

c) Selection of appropriate necessary implants depending upon the bone quality

Appropriate antibiotics and pre-anaesthetic medications were given

\section{Surgical procedure}

Difficulties of exposure with subvastus approach made surgeons to develop minimally invasive midvastus approach, which is a muscle splitting approach first described by Engh in 1997. The minimally invasive midvastus muscle-splitting approach is performed through an anterior midline skin incision. The incision is carried through subcutaneous tissue and deep fascia to expose the quadriceps musculature. The vastus medialis is identified and split full thickness, parallel to its muscle fibres by blunt dissection (Figure 1). The incision is extended to the superior medial corner of the patella and distally along the medial patella and patellar tendon to the level of the tibial tubercle or by the requirement of the surgery. The suprapatellar pouch is divided so that the patella can be everted and dislocated laterally (Figure 2-4).

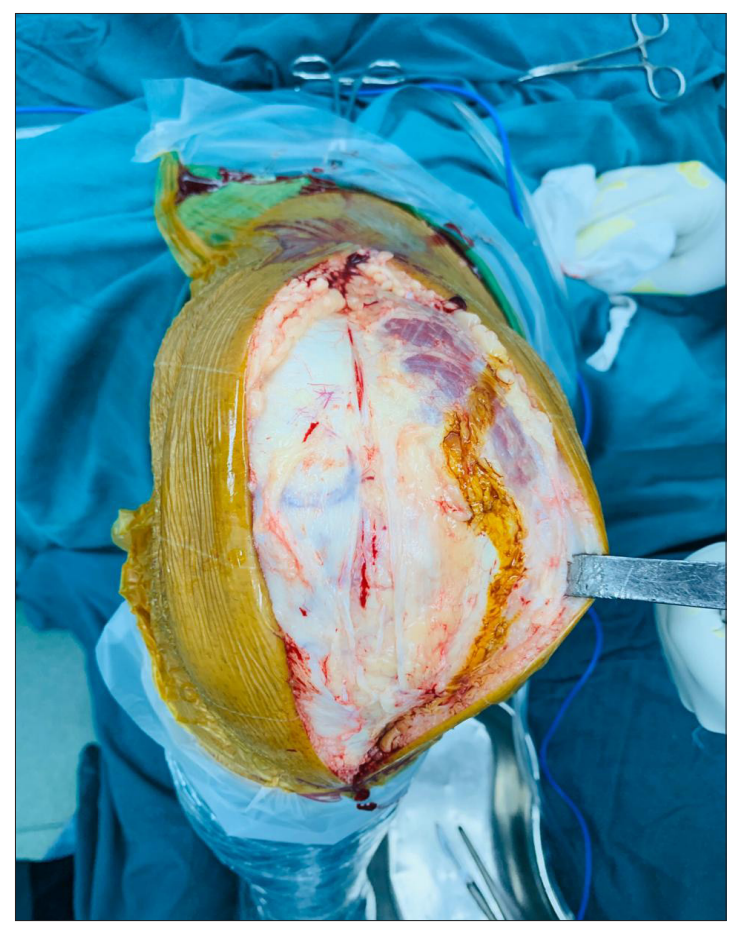

Figure 1: Incision after the midline approach. 


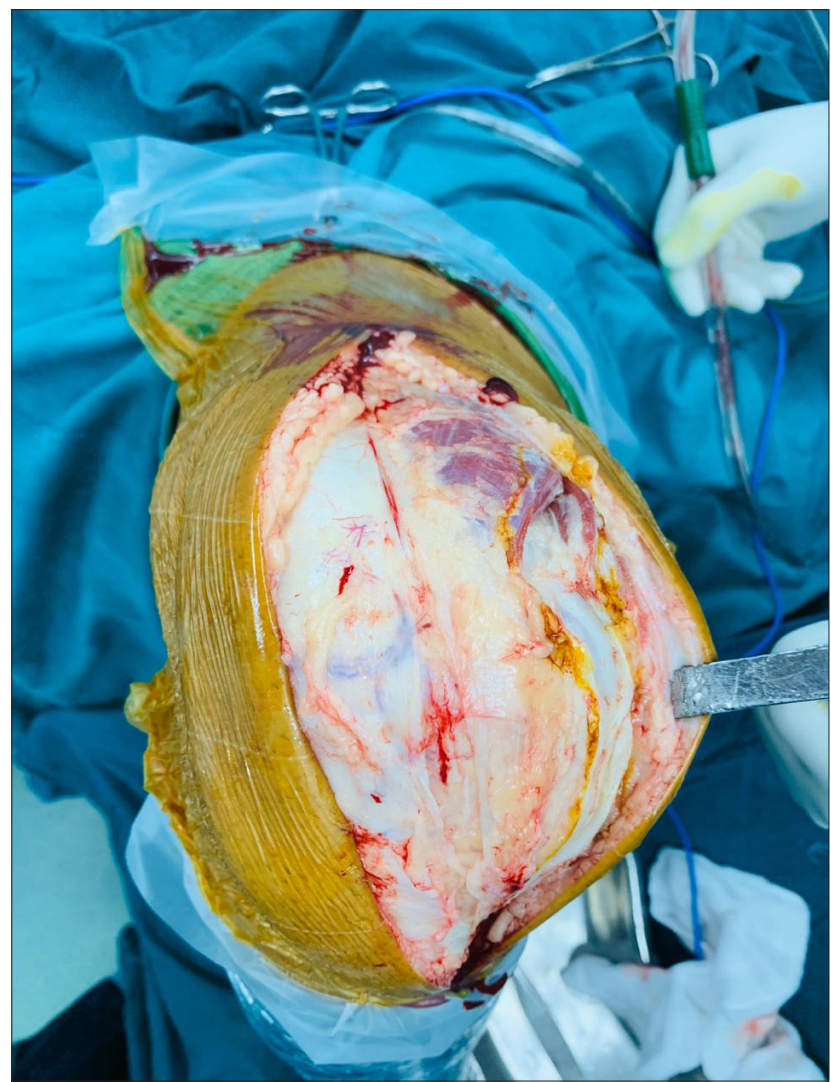

Figure 2: Arthrotomy with the midvastus approach.

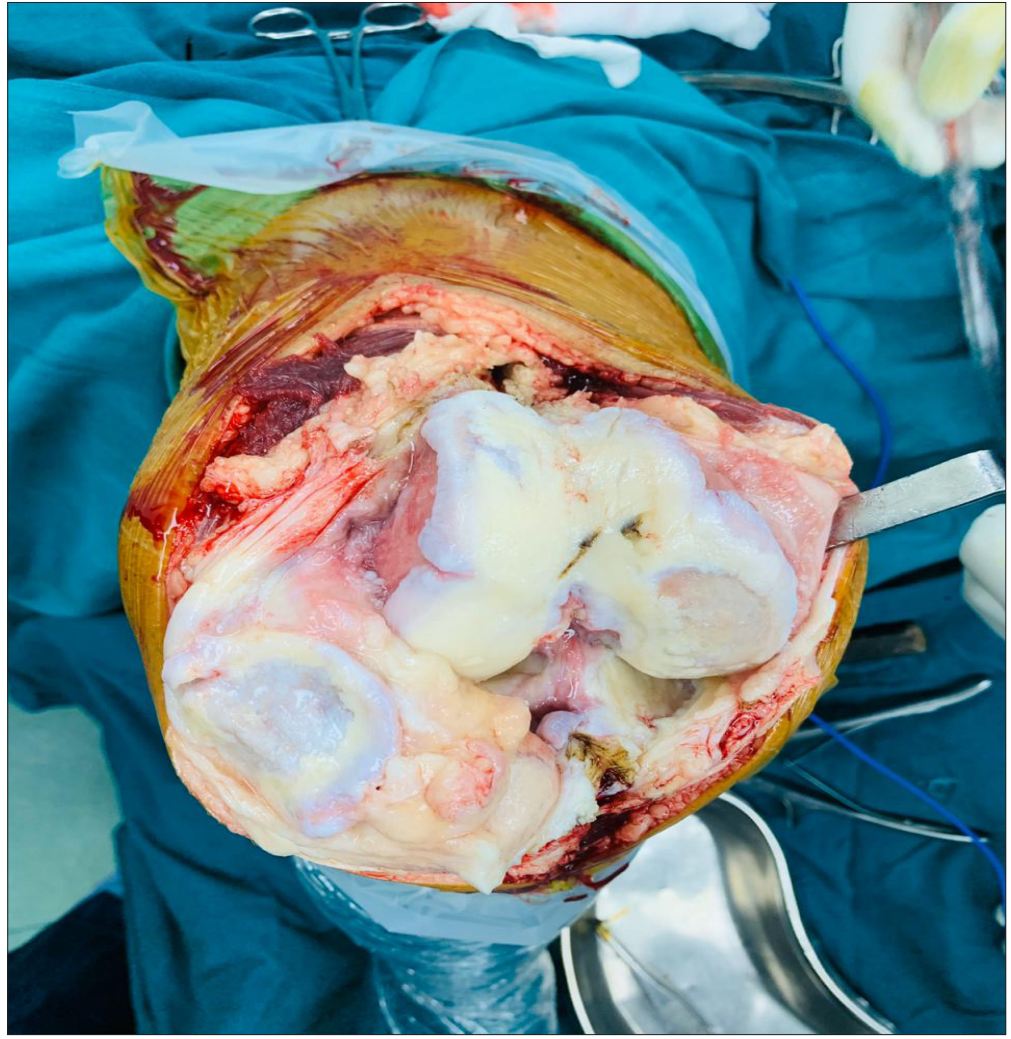

Figure 3: Dislocation of the knee joint with patellar eversion. 


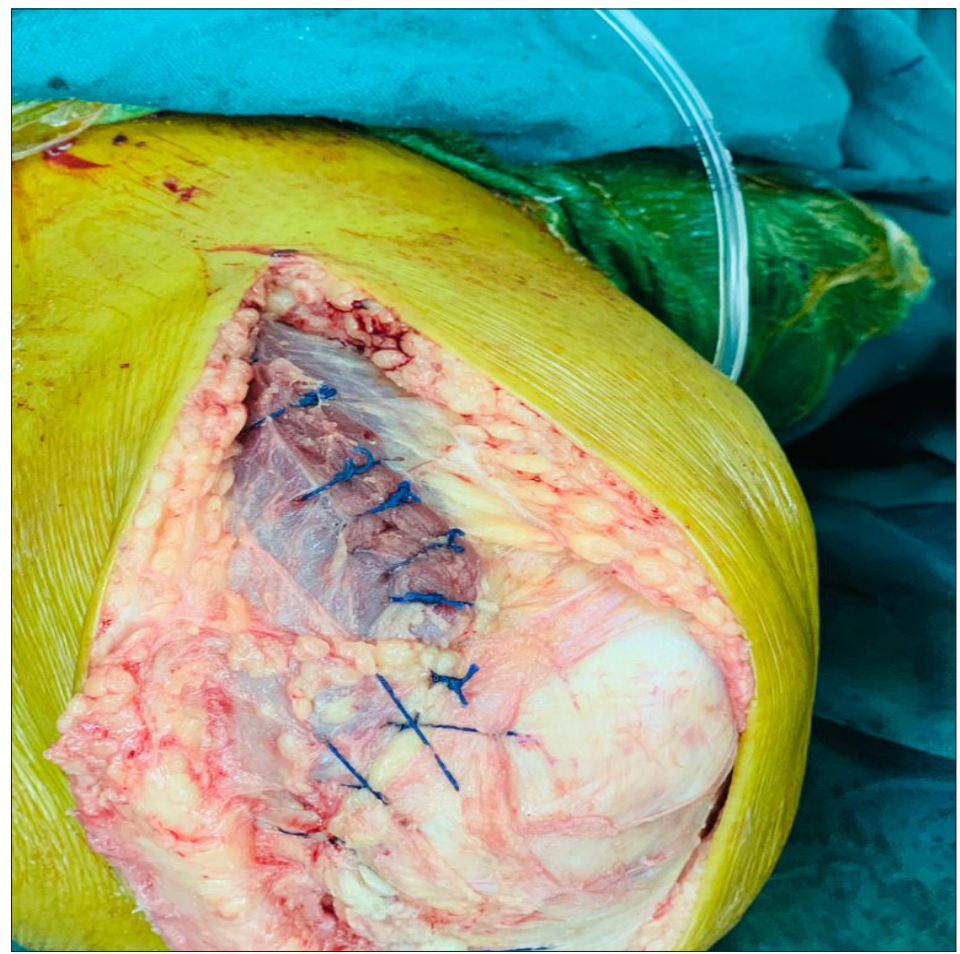

Figure 4: With complete closure after implantation.

\section{Post-operative rehabilitation}

Knee ROM started with drain removal on POD-1

Walking started on POD-1

Post op dressing with check x ray was taken on POD - 2

Bathroom training on day 2

Patient discharged on day 4

Staples were removed on day 12

Patient was followed up for at $1 \mathrm{month}, 3 \mathrm{month}$, and $6 \mathrm{month}$

\section{Results}

\section{a) Age distribution}

Most of patients fall in the age group between 51 to 60 age group,

Table 1:

\begin{tabular}{|c|c|}
\hline Age & Number of Knees \\
\hline $41-50$ & 11 \\
\hline $51-60$ & 35 \\
\hline $61-70$ & 40 \\
\hline $71-80$ & 14 \\
\hline
\end{tabular}

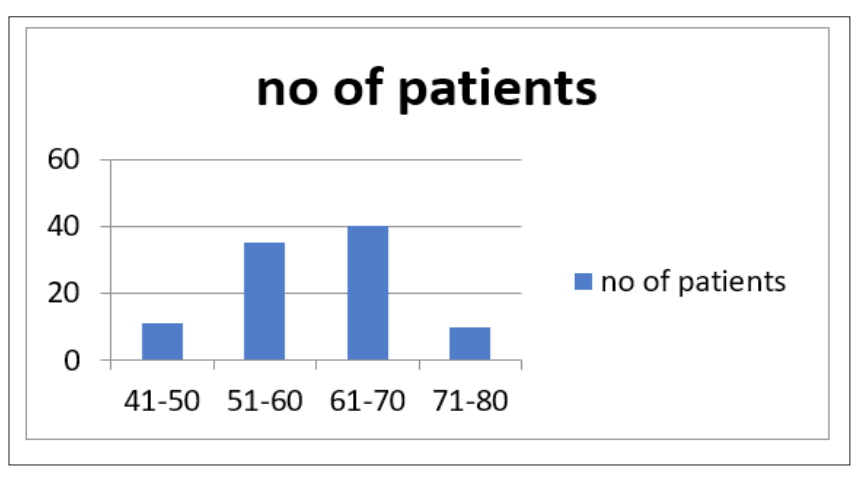

Figure 5:

b) Sex distribution

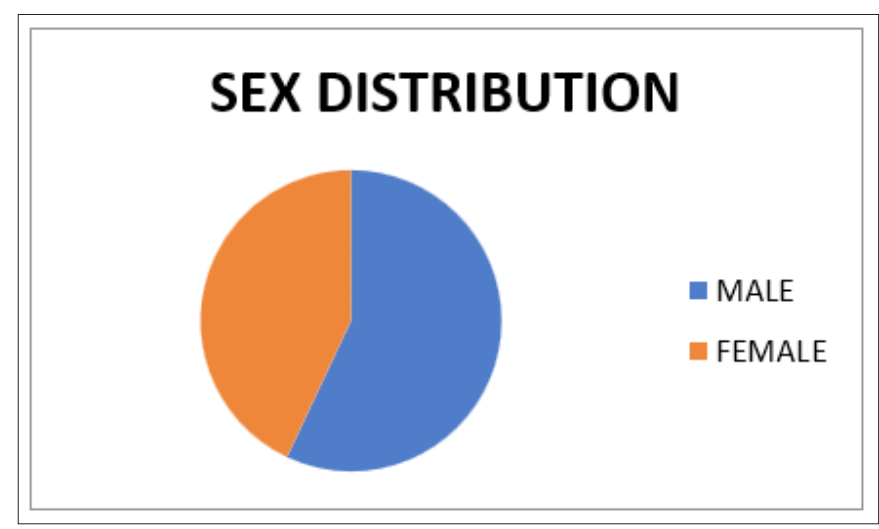

Figure 6: 
Among total no of patients $57 \%$ were males and $43 \%$ were females

c) The no of bilateral cases was 20 compared to 80 unilateral cases

d) The mean operation time was 80 minutes (range 70 to 110 minutes).

e) The mean amount of postoperative hemorrhagic drainage was 200ml (range 100-500ml)

Table 2:

\begin{tabular}{|c|c|c|}
\hline Knee Society Score & Mean & Std. Deviation \\
\hline PreOP_Clinical & 27.8738 & 11.00417 \\
\hline PostOP_Clinical & 92.2816 & 3.87404 \\
\hline PreOP_functional & 54.2233 & 23.43909 \\
\hline PostOp_functional & 94.0777 & 7.72529 \\
\hline
\end{tabular}

f) Preoperative and postoperative comparison of knee society scores: Mean clinical outcome at preoperative period was $27.8 \pm 11.0$ and in postoperative period was $92.2 \pm 3.8$.

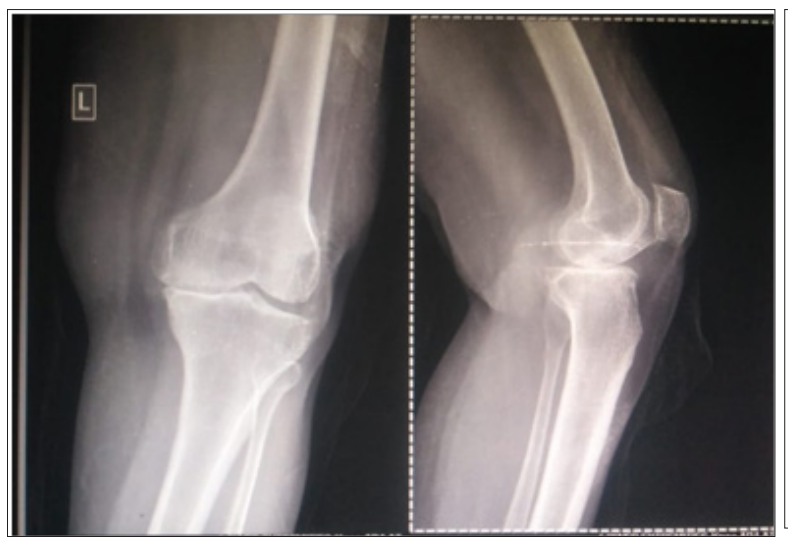

Figure 7: Pre and post op x ray 3rd patient in the study.
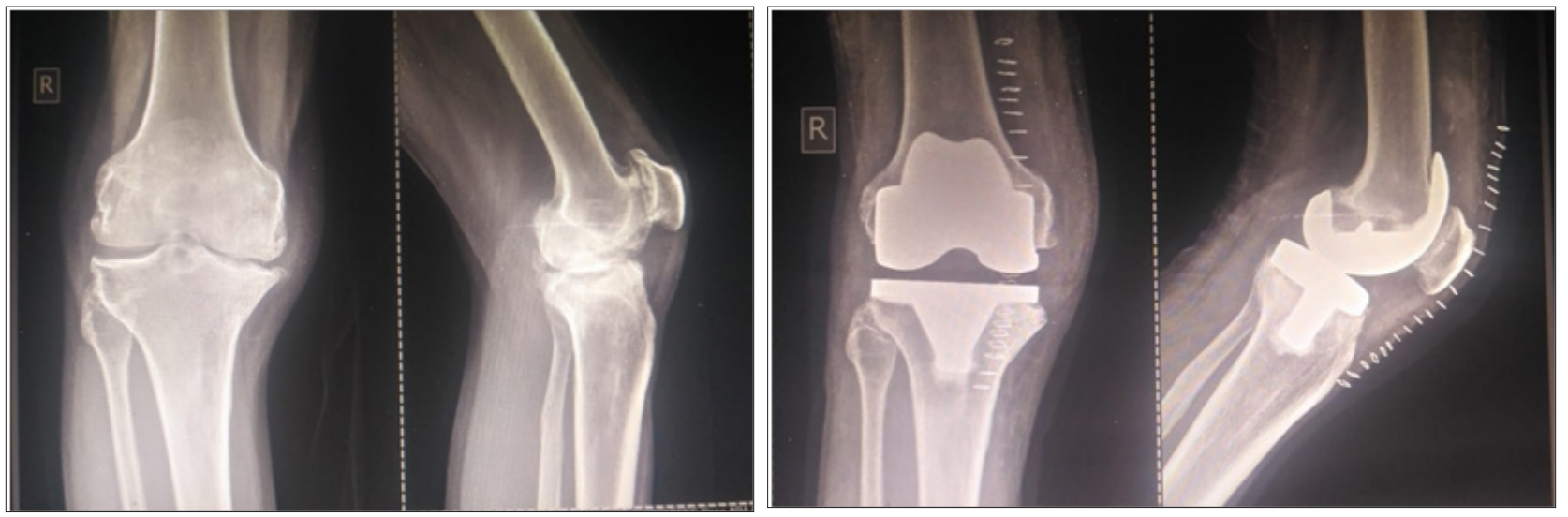

Figure 8: Pre and post op x ray of the 40th patient in the study.

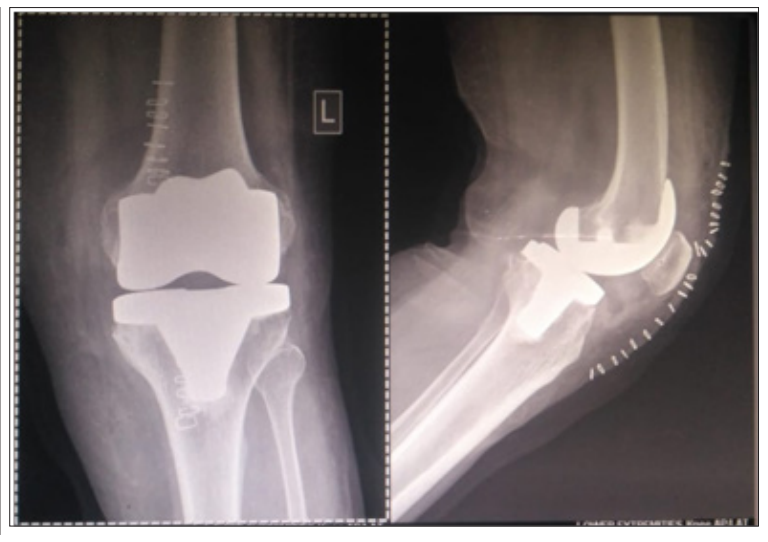

Similarly mean functional outcome at preoperative period was $54.2 \pm 23.4$ and in postoperative period was $94.07 \pm 7.72$. There was significant improvement in clinical and functional outcome after surgery within 2 months of post-operative period (Table 2).

g) Range of movements: Average range of movements in preoperative period was $56.2 \pm 22.7$ and during postoperative period was $111 \pm 9.9$. Hence there was significant increase in average range of movements before and after surgery (Table 3).

\section{Table 3:}

\begin{tabular}{|c|c|c|}
\hline \multirow{2}{*}{} & \multicolumn{2}{|c|}{ Diagnosis } \\
\cline { 2 - 3 } & Mean & SD \\
\hline Pre op ROM & 56.2 & 22.7 \\
\hline Post op ROM & 111.0 & 9.9 \\
\hline P value & \multicolumn{2}{|c|}{$<0.001^{*}$} \\
\hline
\end{tabular}

h) Pre op and post op radiographs and clinical pictures:

(Figure 7 \& 8) 


\section{Discussion}

Total knee arthroplasty main aim is to achieve a painless, stable, and functional joint, which is closely related to the surgical technique [1-3]. Medial parapatellar approach is generally the standard surgical approach in TKA, it allows the patella to evert, in addition to providing integrity of the extensor mechanism [1,4-7]. However, in the medial parapatellar surgical approach, the vastus medialis separated from the patella with an intratendinous incision, resulting in the separation of the descending genicular artery and medial genicular artery from the patella, both of which provide blood flow to the patella [6,8-10]. Moreover, it has been reported that additional problems can arise in parapatellar closure due to the separation of the vastus medialis, and that an abnormal patellar tracking may ensue [5]. A stable patellar tracking is mandatory for a satisfactory outcome in TKA with or without the use of a patellar component [11]. Abnormal patellar tracking can cause severe complications such as patellar dislocation, component erosion, patella fracture, and soft tissue compression $[12,13]$.

If a lateral drifting of the patella is observed intra-operatively, a patellofemoral mismatch is confirmed using the no-thumb test and can be corrected by LRR [11-13]. However, LRR reduces blood flow to the patella and delay in wound healing [14-16]

Muscle atrophy can also be considered among the disadvantages of LRR. Thus, there has been an increasing interest in the subvastus approach defined by Hofman et al. [17] and the midvastus approach defined by Engh et al. [4] for preservation of a steady quadriceps mechanism.

Hofman et al. [17] reported that the subvastus approach provided a good patellar tracking and preservation of the quadriceps mechanism. Fauré et al. [18] found that patellar tilt was less with the subvastus incision compared to the medial parapatellar incision, and the need for LRR was lower. Avascular necrosis of the patella is not seen most cases of subvastus approach, which is regarded as a more anatomical approach [11]. However, the subvastus approach may be associated with postoperative hematoma, muscle ischemia, and difficulties in the lateral eversion of patella due to the medial shift of the arthrotomy distance. Furthermore, separation or the detachment can be seen at the insertion of the patellar tendon $[17,18]$.

The minimally invasive midvastus approach was described by Engh et al. [4] The variation of the medial parapatellar approach to provide a better approach in the knee joint. It is suggested that oblique separation of the muscle fibers of the vastus medialis, there is no much harm to the extensor mechanism in the midvastus approach compared to the medial parapatellar approach. Several studies have favored the minimally invasive midvastus approach over the medial parapatellar approach in terms of ease of application, early rehabilitation, and less pain in the early postoperative period $[2,3,19,20]$.

Engh et al. [4] said that, despite the advantages of the minimally invasive midvastus approach, injuries to neural and vascular structures could occur because of the splitting in the vastus medialis and thus, the procedure should adhere well to the technique. Cooper et al. [21] investigated the relationship between the mid- vastus approach and the proximal popliteal vascular and neural structures supplying the vastus medialis and found that the midvastus approach was safe due to the distance between the patella and the popliteal vessels. Yet, they recommended that the first $4 \mathrm{~cm}$ of the incision beginning from the superior margin of the patella be made with sharp dissection and, subsequently, separation be continued with blunt dissection.

Dalury et al. [22] compared patients undergoing bilateral surgery through the midvastus approach or medial parapatellar approach and found no differences between two groups with respect to the Knee Society scores and function scores, and also found no abnormal electromyographic (EMG) findings associated with the midvastus approach three months post- operatively. In our study, we did not perform EMG studies in the postoperative period, we evaluated our cases with clinical examination and did not find any neurological abnormality.

Bäthis et al. [23] observed early initiation of rehabilitation, less pain, and better proprioception post- operatively in cases of the minimally invasive midvastus approach vs medial parapatellar surgical approach. In our cases, isometric exercises were begun on the first postoperative day and rehabilitation was started under supervision of a physiotherapist following removal of the drain.

Keating et al. [20] compared the midvastus and medial parapatellar surgical approaches in 100 patients of simultaneous bilateral total knee arthroplasty and found no differences between the two groups in terms of the frequency of LRR, range of motion, and straight leg raising capacity of the patients. Hube et al. [24] found excellent or good knee scores in 95\% of 276 knees treated with TKA through the midvastus approach and without the need for LRR. The patella was replaced in all cases and, on postoperative tangential radiographies, maintained its central position in $91 \%$ of the cases.

Kelly et al. [25] reported that LRR was required in one (4.5\%) of 22 cases with the midvastus approach, and in 13 (45\%) of 29 cases with the medial parapatellar approach. However, they detected abnormal EMG changes six months postoperatively in nine cases in the midvastus group, of which seven cases had normal, and two cases had subclinical EMG findings at the end of a five-year followup. The lack of intraoperative LRR need was considered an issue of satisfaction in our series.

In the present study, the mean knee and function scores were found as 92.2 and 94.0, respectively, according to the Knee Society score [26]. The discrepancy between the knee and function scores might result from the relatively high mean age of the patient group (mean age 61.3 years), and from the lack of TKA for the contralateral knee at the final follow-up of patients having bilateral osteoarthritis. This was manifest by the fact that, among patients with bilateral $\mathrm{OA}$, the knee function scores were better in patients who had 
undergone bilateral than unilateral TKA. This suggests that both knees of the patients with bilateral osteoarthritis be operated as early as possible, and if possible, simultaneously, to obtain higher knee function scores.

Despite limitations of this study, including prospective and non-comparative design and short follow-up, we believe that it would contribute to benefits expected from the minimally invasive midvastus approach for surgeons performing total knee arthroplasty.

Prospective and comparative studies on the midvastus approach, reporting mid- and long-term results, with utilization of tangential patella radiographs will definitely provide more conclusive data. In addition, comprehensive studies including gait analysis for evaluation of possible weakening of the quadriceps muscle or myopathy may also be required.

\section{References}

1. Ankle and knee arthroplasty (2007) In: Canale ST (Ed.), Campbell's operative orthopedics. Printing Turkish edition. Hayat Medical Bookstore, Istanbul, Turkey, 1: 243-314.

2. Berth A, Urbach D, Neumann W, Awiszus F (2007) Strength and voluntary activation of quadriceps femoris muscle in total knee arthroplasty with midvastus and subvastus approaches. J Arthroplasty 22: 83-88.

3. Dalury DF, Jiranek WA (1999) A comparison of the midvastus and paramedian approaches for total knee arthroplasty. J Arthroplasty 14: 33-37.

4. Engh GA, Holt BT, Parks NL (1997) A midvastus muscle-split- ting approach for total knee arthroplasty. J Arthroplasty 12: 322-331.

5. Roysam GS, Oakley MJ (2001) Subvastus approach for total knee arthroplasty: a prospective, randomized, and observer- blinded trial. J Arthroplasty 16: 454-457.

6. Cementless total knee arthroplasty (2007) In: Scuderi GR, Tria AJ (Eds.), Knee arthroplasty manual. Techniques in total knee and revision arthroplasty. Hayat Tıp Kitapçllk, Istanbul, Turkey, pp. 80103.

7. Cila E, Güzel V, Özalay M, Tan J, Șimșek SA, et al. (2002) Subvastus versus medial parapatellar approach in total knee arthroplasty. Arch Orthop Trauma Surg 122: 65-68.

8. Kayler DE, Lyttle D (1988) Surgical interruption of patellar blood supply by total knee arthroplasty. Clin Orthop Relat Res (229): 221227.

9. Holtby RM, Grosso P 91996) Osteonecrosis and resorption of the patella after total knee replacement: A case report. Clin Or- thop Relat Res (328): 155-158.

10. Brick GW, Scott RD (1988) The patellofemoral component of total knee arthroplasty. Clin Orthop Relat Res (231): 163-178.

11. Ogata K, Ishinishi T,Hara M 91997) Evaluation of patellar retinacular tension during total knee arthroplasty. Special em- phasis on lateral retinacular release. J Arthroplasty 12: 651-656.

12. Merkow RL, Soudry M, Insall JN (1985) Patellar dislocation following total knee replacement. J Bone Joint Surg 67:1321-1327.

13. Özkoc G, Hersekli MA, Akpınar S, Özalay M, Uysal M, et al. (2005)Time dependent changes in patellar tracking with medial parapatellar and midvastus approaches. Knee Surg Sports Traumatol Arthrosc 13: 654-657.

14. Rosenberg AG, Barden RM, Galante JO (1990) Cemented and ingrowth fixation of the miller-galante prosthesis. Clinical and roentgenographic comparison after three- to six-year follow-up studies. Clin Orthop Relat Res (260): 71-79.

15. Whiteside LA (1996) Fixation for primary total knee arthroplasty: cementless. J Arthroplasty 11: 125-127.

16. Johnson DP, Eastwood DM (1992) Lateral patellar release in knee arthroplasty. Effect on wound healing. J Arthroplasty 7 (Suppl 1): 427 431.

17. Hofmann AA, Plaster RL, Murdock LE (1991) Subvastus (Southern) approach for primary total knee arthroplasty. Clin Or- thop Relat Res (269): 70-77.

18. Fauré BT, Benjamin JB, Lindsey B, Volz RG, Schutte D (1993) Comparison of the subvastus and paramedian surgical approaches in bilateral knee arthroplasty. J Arthroplasty 8: 511-516.

19. White RE Jr,Allman JK, Trauger JA, Dales BH (1999) Clinical comparison of the midvastus and medial parapatellar surgi- cal approaches. Clin Orthop Relat Res (367): 117-122.

20. Keating EM, Faris PM, Meding JB, Ritter MA (1999) Comparison of the midvastus muscle-splitting approach with the median parapatellar approach in total knee arthroplasty. J Arthroplasty 14: 29-32.

21. Cooper RE Jr, Trinidad G, Buck WR (1999) Midvastus approach in total knee arthroplasty: a description and a cadaveric study determining the distance of the popliteal artery from the patellar margin of the incision. J Arthroplasty 14: 505-508.

22. Dalury DF, Snow RG, Adams MJ (2008) Electromyographic evaluation of the midvastus approach. J Arthroplasty 23: 136-40.

23. Bäthis H, Perlick L, Blum C, Lüring C, Perlick C, et al. (2005) Midvastus approach in total knee arthroplasty: a random- ized, double-blinded study on early rehabilitation. Knee Surg Sports Traumatol Arthrosc 13: $545-550$

24. Hube R, Sotereanos N, Reichel H (2002) The midvastus approach for total knee arthroplasty. Orthop Traumatol 10: 235-244.

25. Kelly MJ, Rumi MN, Kothari M, Parentis MA, Bailey KJ, et al. (2007) Comparison of the vastus-splitting and median parapatellar approaches for primary total knee arthroplasty: a prospective, randomized study. Surgical technique. J Bone Joint Surg Am 89 Suppl 2 Pt.1: 80-92.

26. Şener N, Altıntaş F, Beksaç B, Atay E (2003) Comparison of cases in which aggressive teloblast and patellar component were applied in total knee arthroplasty. In: XVIII. National Turkish Orthopedics and Traumatology Congress-Congress Book; 18-23 October 2003; Istanbul Turkey. Turgut Publishing, Istanbul, Turkey, p. 245. 